\title{
Spontaneous Recurrent Absence Seizure-like Events in Wild-Caught Rats
}

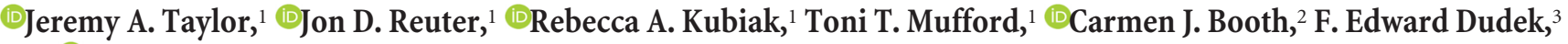 \\ and $\oplus^{\circ D a n i e l ~ S . ~ B a r t h ~}{ }^{1}$ \\ ${ }^{1}$ Department of Psychology and Neuroscience, University of Colorado, Boulder, Colorado 80309, 2Department of Comparative Medicine, Yale University \\ School of Medicine, New Haven, Connecticut 06520, and '3Department of Neurosurgery, University of Utah School of Medicine, Salt Lake City, Utah 84108
}

\begin{abstract}
Absence epilepsy is a heritable human neurological disorder characterized by brief nonconvulsive seizures with behavioral arrest, moderate-to-severe loss of consciousness (absence), and distinct spike-wave discharges (SWDs) in the EEG and electrocorticogram (ECoG). Genetic models of this disorder have been created by selectively inbreeding rats for absence seizure-like events with similar electrical and behavioral characteristics. However, these events are also common in outbred laboratory rats, raising concerns about whether SWD/immobility accurately reflects absence epilepsy as opposed to "normal" rodent behavior. We hypothesized that, if SWD/ immobility models absence seizures, it would not exist in wild-caught rats due to the pressures of natural selection. To test this hypothesis, we compared chronic video/electrocorticogram recordings from male and female wild-caught (Brown-Norway [BN]) rats to recordings from laboratory outbred BN, outbred Long-Evans, and inbred WAG/Rij rats (i.e., a model of absence epilepsy). Wild-caught BN rats displayed absence-like SWD/immobility events that were highly similar to outbred BN rats in terms of spike-wave morphology, frequency, diurnal rhythmicity, associated immobility, and sensitivity to the anti-absence drug, ethosuximide; however, SWD bursts were less frequent and of shorter duration in wild-caught and outbred BN rats than the outbred Long-Evans and inbred WAG/Rij strains. We conclude that SWD/immobility in rats does not represent absence seizures, although they appear to have many similarities. In wild rats, SWD/immobility appears to represent normal brain activity that does not reduce survival in natural environments, a conclusion that logically extends to outbred laboratory rats and possibly to those that have been inbred to model absence epilepsy.
\end{abstract}

Key words: absence; epilepsy; SWD

\section{Significance Statement}

Spike-wave discharges (SWDs), behavioral arrest, and diminished consciousness are cardinal signs of seizures in human absence epilepsy and are used to model this disorder in inbred rats. These characteristics, however, are routinely found in outbred laboratory rats, leading to debate on whether SWD/immobility is a valid model of absence seizures. The SWD/immobility events in wild-caught rats appear equivalent to those found in outbred and inbred rat strains, except for lower incidence and shorter durations. Our results indicate that the electrophysiological and behavioral characteristics of events underlying hypothetical absence epilepsy in rodent models are found in wild rats captured in their natural environment. Other criteria beyond observation of SWDs and associated immobility are required to objectively establish absence epilepsy in rat models.

\section{Introduction}

Childhood absence epilepsy is an idiopathic, nonconvulsive disorder with a genetic basis (Crunelli and Leresche, 2002). Absence

\footnotetext{
Received May 8, 2018; revised March 9, 2019; accepted March 27, 2019

Author contributions: J.A.T. and D.S.B. designed research; J.A.T., J.D.R., R.A.K., T.T.M., C.J.B., and D.S.B. performed research; J.A.T., R.A.K., and D.S.B. analyzed data; J.A.T. and D.S.B. wrote the first draft of the paper; J.A.T., J.D.R., R.A.K., C.J.B., F.E.D., and D.S.B. edited the paper; D.S.B. wrote the paper.

This work was supported by the U.S. Army Medical Research and Material Command Grant PR100040 and EP150033, and Citizens United for Research in Epilepsy CURE Grant 7153.

F.E.D. has received consulting fees from Rugen Biomedical and Neurona Therapeutics, and has equity interest in and receives consultant fees from Epitel, Inc., which is a company that makes telemetric recording devices; however, this work did not use these devices. The remaining authors declare no competing financial interests.

Correspondence should be addressed to Daniel S. Barth at daniel.barth@colorado.edu.
}

seizures are typically a childhood disorder (Panayiotopoulos, 2001; Posner, 2013) and are characteristically brief (several seconds), often with behavioral interruption and mild-to-severe impairment of consciousness (Blumenfeld, 2005, 2012). One of the most distinctive electrographic features of absence seizures are synchronous "spike-wave" discharges (SWDs) occurring at $\sim 3$ $\mathrm{Hz}$ and recorded across the cerebral cortex (Smith, 2005).

Inbreeding for several decades has generated strains of rats that have been used to model absence epilepsy. Specifically, the

https://doi.org/10.1523/JNEUROSCl.1167-18.2019

Copyright $\odot 2019$ the authors 
WAG/Rij strain, originating initially from the Wistar Albino Glaxo Laboratories (Coenen and Van Luijtelaar, 2003; Booth et al., 2010; Sarkisova and Van Luijtelaar, 2011), and the Genetic Absence Epilepsy Rat strain from Strasbourg (GAERS), Germany (Marescaux et al., 1992), are both well-accepted genetic animal models of absence epilepsy used for antiseizure drug discovery and examination of underlying seizure mechanisms. In addition to a genetic origin, these models appear to approximate human absence seizures, including brief (seconds) SWDs in the electrocorticogram (ECoG)/EEG, associated immobility (i.e., behavioral interruption), and possible impairment of consciousness.

The cardinal traits of absence seizures in rat models, specifically stereotyped SWD/immobility events, have also been recognized in most outbred laboratory rat strains (Sarkisova and Van Luijtelaar, 2011). The presence of SWD/immobility in outbred strains raises several important questions. Do most laboratory rats used in behavioral experiments suffer from undetected absence epilepsy? Has selective breeding to maintain stable behavioral and physiological traits in these commercial colonies inadvertently resulted in introducing and/or preserving the genes responsible for absence seizures? Or are SWD/immobility events normal rat behavior, and, if so, by what criteria can they then be judged abnormal in inbred rat colonies?

Whether or not SWD/immobility reflects absence seizures in outbred rats has a long history of controversy (Kaplan, 1985; Wiest and Nicolelis, 2003; Shaw, 2004; Pearce et al., 2014). We recently found that Sprague Dawley and Long-Evans (LE) rats displayed frequent SWD/immobility events that appeared to be absence-like seizures very similar to inbred WAG/Rij absence epilepsy rats (Taylor et al., 2017). We concluded that, although these events could model mild absence epilepsy with only partial attenuation of consciousness, they may instead be a form of modifiable behavior. This was due to observation that the rats shortened the duration of SWD bursts followed by preemptively checking for a delayed reward. Whether SWD/immobility events are absence-like seizures or not, they are nonetheless present in commercial outbred laboratory rat strains commonly used in multiple areas of research. Therefore, determining whether these events are pathophysiological is critical. While spontaneous and repeated, albeit partial, loss of consciousness and immobility would still be a major confound for behavioral research using outbred rats, momentary loss of consciousness with spontaneous and involuntary immobility could have substantial negative consequences in natural environments with predators. In the present study, we hypothesized that, if SWD/immobility events found in outbred strains of laboratory rats reflected absence-like seizures, as opposed to normal behavior, then they would be absent in rats captured in the wild. Heritable pathophysiological events (e.g., SWDs) would seem to be disadvantageous and would presumably be eliminated by natural selection in wild rats unless, perhaps, the thalamocortical oscillations underlying SWDs are beneficial for survival (e.g., for sensory processing or signal detection) despite possible accompanying behavioral disruption. To test this hypothesis, we performed chronic video/ECoG recording of wild-caught $\mathrm{BN}$ rats to search for any SWDs and their associated behavior. We compared these results with similar recordings of outbred BN rats, outbred LE rats commonly used in behavioral studies, and inbred WAG/Rij rats that have served as a model of absence epilepsy.

\section{Materials and Methods}

Ten wild Rattus norvegicus ( 5 male, 5 female; similar to Brown Norway $[\mathrm{BN}]$ rats) were trapped in Boulder, $\mathrm{CO}$, at different locations separated by several miles, over approximately a year's time during the summer and fall seasons when nighttime temperatures did not fall below $4^{\circ} \mathrm{C}$. Traps were set in the evening (16:00-17:00 h) and checked at dawn (06:0007:00 h). A total of 8 rats were captured along the Boulder Creek: June 3-11, 2017, June 28, 2017, and October 30, 2017 (map coordinates: $\left.40^{\circ} 00^{\prime} 26.7^{\prime \prime} \mathrm{N} 105^{\circ} 14^{\prime} 58.6^{\prime \prime} \mathrm{W}\right), 4$ on June $15,2018\left(40^{\circ} 01^{\prime} 31.0^{\prime \prime} \mathrm{N}\right.$ $\left.105^{\circ} 14^{\prime} 48.0^{\prime \prime} \mathrm{W}\right)$, and 1 on June $25,2018\left(40^{\circ} 01^{\prime} 17^{\prime \prime} \mathrm{N} 105^{\circ} 12^{\prime} 55.2^{\prime \prime} \mathrm{W}\right)$, and an additional 2 rats were captured on October 13, 2018 at the Western Disposal Service dump. All 10 wild rats were placed in quarantine for the duration of the study. Health of the rats was assessed serologically and via PCR using the Idexx Radil Advantage Global health monitoring panel. Initially, 3 rats were treated for endoparasites and ectoparasites before instrumentation for chronic video/ECoG recording, using a combination of selamectin topically, $15 \mathrm{mg}$ at day 0 and day 30, Droncit (praziquantel) $5 \mathrm{mg} / \mathrm{kq}$ (s.c.), and metronidazole $10 \mathrm{mg} / \mathrm{kg}$ (p.o.) $\times 5 \mathrm{~d}$. After considering the possibility that this treatment could influence our findings and conclusions about wild rats in relation to SWDs, we decided to withhold this treatment on all rats captured thereafter $(n=7)$. However, because it was not possible to instrument rats in the wild, we could not assess or control for the potential influence(s) of the indoor environment and nutritional/dietary changes on SWDs. Although the age of the rats could not be determined, their weight $(270.7 \pm 24.6 \mathrm{~g})$ approximated that of 4 months old commercially bred BN rats. For comparison with the wild rats, healthy young ( 4 months old; $n=6 ; 270.8 \pm 3.9 \mathrm{~g}$ ) and older ( 10 months old; $n=6 ; 368.3 \pm 10.5 \mathrm{~g}$ ) male BN rats (Envigo), male (10 months old) LE rats ( $n=5$, Envigo), and inbred female WAG/ RijCmcr (WAG/Rij) rats (10 months old; $n=6$; Department of Comparative Medicine, Yale University School of Medicine, New Haven, CT) were also studied. Wild rats in quarantine, as well as the domestic BN, LE, and WAG/Rij rats, were housed under standard laboratory conditions in a temperature- and relative humidity-controlled (i.e., $20 \pm 1^{\circ} \mathrm{C}$ and $40 \%$, respectively) environment with a normal $12 \mathrm{~h}$ light-dark cycle. Sterile irradiated food and reverse osmosis water were available ad libitum. All procedures were performed in accordance with University of Colorado Institutional Animal Care and Use Committee guidelines for the humane use of laboratory rats in biological research.

Surgery and video/ECoG recording. Epidural stainless-steel screw electrodes (E363-20 Elect W, $3.2 \mathrm{~mm}$ screw, $20 \mathrm{~mm}$ length wire; Plastics One, Medical Design and Manufacture) were implanted using aseptic surgical procedures. At the start of surgery, rats were anesthetized with isoflurane (2.5\%; Fluriso, isoflurane, USP, VETone) gas anesthesia. A subcutaneous saline bolus $(1 \mathrm{ml} / 100 \mathrm{~g}$; $0.9 \% \mathrm{NaCl}$; sterile saline solution, USP, VETone) was administered, oxygen provided (2 L/min; oxygen, compressed USP, Airgas Healthcare), artificial eye lubricant applied (petrolatum ophthalmic sterile ointment; Puralube Vet Ointment, Dechra Veterinary Products), body temperature maintained with an electric heating pad, and life signs were monitored (heart rate and $\mathrm{Sp} /$ $\mathrm{O}_{2}$ ). A small longitudinal incision over the dorsal portion of the skull was then made (caudal to the eyes and rostral to the ears) effectively exposing the frontal and parietal bones (anteroposterior $4.0-8.5 \mathrm{~mm}$, mediolateral $\pm 5.0 \mathrm{~mm}$ from bregma). Fascia and blood were cleared from the skull and the surface allowed to dry (hemostasis achieved with $\sim 1 \mathrm{ml}$ of epinephrine dripped over the area, $1 \mathrm{mg} / \mathrm{ml}$; VETone). Burr holes were drilled through the skull with care taken not to penetrate the meninges. From bregma, two electrode screws were placed over the approximate orofacial region of the somatosensory cortex (anteroposterior $-2.0 \mathrm{~mm}$, mediolateral $\pm 4.0 \mathrm{~mm}$ ), along with one reference electrode screw (anteroposterior $3.0 \mathrm{~mm}$, mediolateral $1.0 \mathrm{~mm}$ ), one ground electrode screw (anteroposterior $-7.5 \mathrm{~mm}$, mediolateral $4.0 \mathrm{~mm}$ ), and two anchor screws (anteroposterior $3.0 \mathrm{~mm}$, mediolateral $-1.0 \mathrm{~mm}$; anteroposterior $-7.5 \mathrm{~mm}$, mediolateral $-4.0 \mathrm{~mm}$ ). Electrode wires and a round six socket polyamide Electrode Pedestal (Plastics One) were cemented in place with a mixture of dental cement powder, dental liquid, and antibiotic powder (Uniprim, Macleod Pharmaceuticals) (ratio of $1.8 \mathrm{~g}: 2 \mathrm{ml}: 0.2$ g, respectively), which was kept cool by continuously drizzling sterile saline $\left(\sim 23^{\circ} \mathrm{C}\right)$ over the dental cement head-cap while it hardened. Rats received an intramuscular injection of Buprenex $(0.05 \mathrm{ml} / 100 \mathrm{~g}$; buprenorphine hydrochloride, Par Pharmaceuticals) after completion of the surgery. Rats were given 1 week of recovery with soft food diet gel 
available (DietGel Recovery, Purified Dietary Supplement, Clear $\mathrm{H}_{2} \mathrm{O}$ ) before video/ECoG recording began. For video/ECoG recording, animals were tethered to an electrode harness (Plastics One, 363) and slipring commutator (Plastics One, SL6C) permitting free movement for ECoG monitoring throughout the experiment. ECoG signals were amplified $(\times 10,000)$, analog filtered $(1-200 \mathrm{~Hz}$ bandpass), and digitized at $500 \mathrm{~Hz}$. Recording was performed for 5-30 d.

Supervised pattern recognition of SWDs. Similar to our previous studies (Rodgers et al., 2015; Taylor et al., 2017), automated detection was used to quantify SWD events in the ECoG data. For a given rat, SWDs were visually identified, the peaks of 30-50 spikes were digitally marked, and $1 \mathrm{~s}$ samples surrounding each peak were extracted (see Fig. $2 A$ ) and averaged to construct an SWD spike template (see Fig. $2 B$ ). The template was time-shifted by $2 \mathrm{~ms}$ increments in relation to several preidentified SWD bursts (see Fig. 2C) and the cross-covariance computed at each shift (see Fig. 2D). A threshold was then visually set for the crosscovariance beyond which candidate SWD spikes were detected (see Fig. $2 D$, black trace). Approximately 100 candidate spikes were extracted from EEG samples with known SWDs and those with representative noise (including slow-wave sleep) and artifact, and used to train a Support Vector Machine (SVM) (Nandan et al., 2010; Orrù et al., 2012) to automatically discriminate between SWDs and noise. The trained SVM model for a given rat was then tested with raw data in that animal to visually identify and label correct and incorrect SWD identifications. These were used to train a second SVM model. The "two-stage" SVM model was then used to extract SWDs in that animal for all subsequent recording. Finally, SWD burst lengths were computed by examining interspike intervals. Spikes with intervals $<1 \mathrm{~s}$ were considered to be part of the same burst, to account for waxing and waning of SWD amplitude within bursts (where the SVM may skip several spikes due to low amplitude). Additionally, spikes with intervals $<66 \mathrm{~ms}$ were rejected to eliminate artifacts due to multiple triggering on the same spike (SWD frequencies are typically $7-10 \mathrm{~Hz}$, so the $66 \mathrm{~ms}$ interval threshold set a maximum of $15 \mathrm{~Hz}$ beyond which spikes were rejected).

Power spectrum modeling. To compare the spectral frequency distribution of SWDs between rats and strains, power spectra of $1 \mathrm{~s}$ samples identified with SVM were computed using a Welch transform and averaged across $\sim 100$ SWDs for a given rat (MATLAB Signal Processing Toolbox, The MathWorks).

Diurnal rhythmicity of SWD spikes. To examine differences in SWDs during lights-on versus lights-off periods, we first computed spike counts during SWDs (regardless of burst lengths) across a $24 \mathrm{~h}$ period (in $30 \mathrm{~m}$ bins), accumulating results over multiday epochs for each rat. All data were scaled (range $=0-100$ ) because there were substantial differences in overall spike counts between rats. Scaling data prevented rats with large spike counts from dominating the results. Counts were then averaged across the lights-on (06:00-18:00 h) versus lights-off (18:00-06:00 h) periods.

We also examined diurnal changes in SWDs by summing spike counts across $24 \mathrm{~h}$ and expressing counts for each $30 \mathrm{~m}$ bin as the percentage of this total, separately for each rat. We refer to "diurnal" as opposed to "circadian" because we did not examine the relative influence of endogenous versus environmental (i.e., light-dark, feeding, etc.) factors. Results were averaged across rats within each strain. Single-component Cosinor analysis (Refinetti et al., 2007) was then used to examine possible diurnal rhythms in the SWD spike counts. This simple method is common in analysis of diurnal and circadian rhythms and consists of fitting a single cosine curve with a $24 \mathrm{~h}$ period to the data. The phase of the maximum in relation to a fixed reference time (midnight or $0 \mathrm{~h}$ ) is known as the "acrophase" or "center of gravity" (COG) and gives information concerning the relative timing of diurnal rhythmicity for comparison between animals.

Diurnal rhythmicity of movement. Motion across successive frames of video (recorded simultaneously with the $\mathrm{ECoG}$ ) was quantified using the Lucas-Kanade method of optical flow analysis (Barron et al., 1994) (MATLAB Computer Vision System Toolbox, The MathWorks). Optical flow is a method of motion detection that generates motion vectors in relation to orderly pixel displacements from one video frame to the next (stationary objects result in no pixel displacement). With a camera cali- brated for pixels per meter displacement and known distance of objects, the motion vectors reveal exact distances traveled over time (frame rate) and thus exact velocity. While all of our rats were at a fixed distance from the video cameras with the same resolution and the same frame rate, the cameras themselves were not calibrated. Optical flow magnitudes were therefore scaled (range $=0-100$ ) for each rat. Similar to our analysis of SWD spiking, continuous optical flow magnitudes were divided into $30 \mathrm{~m}$ bins across each $24 \mathrm{~h}$ period and averaged over multiday epochs for each rat. Differences in motion during lights-on $(06: 00-18: 00 \mathrm{~h})$ versus lights-off (18:00 - 06:00 $\mathrm{h}$ ) periods were determined by averaging optical flow within each period. Diurnal changes in motion for each rat were then computed for each $30 \mathrm{~m}$ bin throughout the day as the percentage of total daily flow magnitude.

Movement during SWD bursts. Motion during and between SWD bursts was also quantified from the optical flow of video records recorded simultaneously with ECoG. Fifty blocks (30 m each) of video/ECoG data that contained at least 1 clear SWD burst were examined in each rat. For each block, segments during and between SWD bursts were visually identified and marked for subsequent analysis. Visual identification was used instead of automated SWD detection because it more precisely identified the burst and interburst intervals, particularly against a noisy back ground. Optical flow magnitudes during and between corresponding bursts (see Fig. 9) were scaled (range $=0-100$ ) across all extracted data for a given rat and then separately averaged.

Ethosuximide (ETX) pharmacology. One hour before lights-off (17:00 h), rats were given one dose of ETX (100 mg/kg, E7138, Sigma-Aldrich) dissolved in saline $(0.9 \% \mathrm{NaCl})$ and injected intraperitoneally. The wild rats (and only the wild rats) were briefly anesthetized with isoflurane $(2.5 \%)$ twice: once when transferred $(\sim 10-15 \mathrm{~s}$ anesthetized) from the home-cage to recording chambers (Deep Rock water jug; $26.5 \mathrm{~cm}$ diameter $\times 30 \mathrm{~cm}$ height $)$ and once during injection ( $\sim 60-90 \mathrm{~s}$ anesthetized) due to handling difficulties (wild rats were liable to flee or become aggressive toward the experimenter immediately upon opening their cage). Recording began after observing recovery from anesthesia (i.e., the rat was balanced and coordinated in voluntary movement and responsive to sound (hand-clap), which happened within 2-4 min. To avoid possible residual effects of the anesthesia and allow for an accurate comparison to the domestic rat groups (which were not anesthetized before injection or cage transfer), the first $30 \mathrm{~min}$ of video/ECoG data for all rat groups was excluded from analysis. Video/ECoG data were recorded for $72 \mathrm{~h}$ (broken into $24 \mathrm{~h}$ segments of baseline, ETX, and recovery). The number of seconds of SWDs was counted and summed for each half-hour of video/ ECoG data, which was then averaged for each condition and normalized to baseline SWD values. ETX plasma concentrations tend to peak at $\sim 2 \mathrm{~h}$ after injection and steadily decline thereafter; as such, the first $6 \mathrm{~h}$ (starting at 17:30 h) of each condition was selected for analysis.

Statistical analysis. All results are expressed as mean \pm SEM. One- and two-way ANOVA was used to determine significant within-subject's effects between factors. Post hoc comparisons (Student's $t$ test with a Bonferroni correction for multiple comparisons) were conducted to follow-up significant overall ANOVAs. Data were analyzed using the MATLAB Statistics and Machine Learning Toolbox (The MathWorks); and for circular functions (e.g., time of day), a circular statistics toolbox designed for MATLAB was used (Berens, 2009). In all cases, statistical significance was set at $p \leq 0.05$.

Terminology. Here and elsewhere, an "SWD burst" or "burst" is defined as an epoch of continuous SWD. Bursts can have different durations, and the number of bursts of different durations or the total number of bursts is reflected in the burst counts per day. In contrast, the terms "SWD spikes" or "spike counts" are used to define the number of spikes during all bursts for a given rat or condition. Infrequent and/or short SWD bursts will have lower spike counts than frequent and/or long bursts.

\section{Results}

\section{SWDs in the raw ECoG}

Of the 10 wild rats, 9 displayed spontaneous SWD bursts in their ongoing, continuously recorded video/ECoGs, typically with a duration of $0.5-1 \mathrm{~s}$ (Fig. 1A), but also with bursts $>1 \mathrm{~s}$ (Fig. 1B). 
SWD bursts that contained $<3$ spikes were rejected (e.g., Fig. $1 A$, arrows). Of the 6 weight-matched $\mathrm{BN}$ rats ( 4 months old), 3 had no SWD bursts and the other 3 had very few and short SWD bursts (Fig. 1C). This group was therefore not subjected to further analysis. For the older BN rats (10 months old), 5 of 6 displayed numerous short SWD bursts (Fig. 1D), similar to the wild rats, along with infrequent longer bursts (Fig. 1E). In addition to short bursts, the 5 LE (Fig. $1 F$ ) and 6 WAG/Rij (Fig. 1I) rats had longer bursts of several seconds (Fig. $1 G$ and Fig. 1J, respectively); the main distinction of these later groups compared with both the wild and $\mathrm{BN}$ rats were the presence of much longer bursts, often extending over $10 \mathrm{~s}$ duration (Fig. $1 H$ and Fig. $1 K$, respectively).

\section{SWD power spectra}

SWD features in the raw ECoG were quantified using pattern recognition (Fig. 2) to extract all recorded bursts in a given animal (described in Materials and Methods). SWD bursts in our wild rats displayed a distinct frequency spectrum (common to SWDs reported for most outbred and inbred rats) due to the quasiperiodic waveform, with a fixed fundamental frequency peak in the range of 7-10 Hz, and peaks at whole harmonics of this fundamental frequency (Van Hese et al., 2003, 2009; Shaw, 2004; Sitnikova et al., 2009; Rodgers et al., 2015; Taylor et al., 2017). Figure $3 A$ (blue trace) shows an example of spectral analysis in a wild rat, averaged across all SWD bursts, with a fundamental frequency of $7.5 \mathrm{~Hz}$ and prominent harmonic peaks at $\sim 15$ and $22.5 \mathrm{~Hz}$. This spectral signature was due to the repeating "spike" component of the waveform. In this example, the averaged power spectrum was plotted in $\mathrm{mV}$ instead of $\mathrm{mV}^{2}$ for amplitude comparison to the corresponding averaged SWD time series (Fig. 3B, blue trace) with typical spike and wave components. We then smoothed the spectral coefficients to preserve the fundamental frequency but attenuate the harmonic components (Fig. 3A, red trace) and performed an inverse FFT to re-create the remaining time series. As can be seen in Figure $3 B$, the re-created waveform without spectral harmonics appeared as a simple sinusoidal wave (red trace) without the characteristic spike of the SWD complex (blue trace), that distinguishes it from sleep spindles and other typical brain oscillations (e.g., $\alpha, \mu$, and $\theta$ rhythms), which may be of similar frequency but of sinusoidal wave morphology with no spikes. The fundamental frequency of SWDs in wild rats $(7.5 \pm 0.22 \mathrm{~Hz})$ was similar to the BN, LE, and WAG/Rij groups $(9.1 \pm 0.21,8.5 \pm 0.4$, and $9.6 \pm 0.19 \mathrm{~Hz}$, respectively; Fig. 4). However, while frequency differences between strains were small, one-way ANOVA indicated a main effect of strain $\left(F_{(3,18)}=12.74: p<0.001\right)$. Wild rats were $\sim 1.5 \mathrm{~Hz}$ slower than $\mathrm{BN}$ and WAG/Rij rats $(p=0.003$ and $p=0.006$, respectively). There were no other significant differences in fundamental frequencies between the strains.

\section{SWD burst rates and durations}

Figure 5 shows SWD burst rates (bursts/d) as a function of duration for wild, BN, LE, and WAG/Rij rats. SWD burst rates and durations appeared similar between wild and $\mathrm{BN}$ rats (Fig. 5A,B) and less frequent and shorter than the LE and WAG/Rij rats, which had more bursts/d and more bursts of duration $>2.0 \mathrm{~s}$ (Fig. 5C,D, arrows). Figure $5 E$ summarizes the similarities and differences in average SWD burst durations and burst rates between groups. Wild (dashed red) and $\mathrm{BN}$ (solid red) rats were similar in both burst duration and rate. LE (dashed blue) and WAG/Rij (solid blue) were also similar, with longer and more frequent SWD bursts than the wild and domestic $\mathrm{BN}$ rats. Oneway ANOVA of SWD burst rates revealed a main effect of strain $\left(F_{(3,17)}=137.82: p<0.001\right)$. Average rates for wild rats $(198.7 \pm$ 83.5 bursts/d) did not differ from BN rats $(281 \pm 48.9$ bursts/d) but were significantly less than both LE $(2683.1 \pm 174.6$ bursts/d $)$ and WAG/Rij ( $3380 \pm 180.3$ bursts/d) rats ( $p<0.001$ for both). Rates for BN rats were also significantly less than both LE and WAG/Rij rats ( $p<0.001$ for both), whereas rates did not differ between LE and WAG/Rij animals. One-way ANOVA of SWD burst durations also revealed a main effect of strain $\left(F_{(3,17)}=\right.$ 20.53: $p<0.001$ ). Paired comparisons were similar to those for rates. Average durations for wild rats $(1.25 \pm 0.010 \mathrm{~s})$ did not differ from $\mathrm{BN}$ rats $(1.34 \pm 0.016 \mathrm{~s})$ but were significantly shorter than both $\operatorname{LE}(2.28 \pm 0.014 \mathrm{~s})$ and WAG/Rij $(2.64 \pm 0.015 \mathrm{~s})$ rats $(p<0.001$ for both). Durations for BN rats were also significantly shorter than both LE and WAG/Rij rats $(p=0.046$ and $p=$ 
A
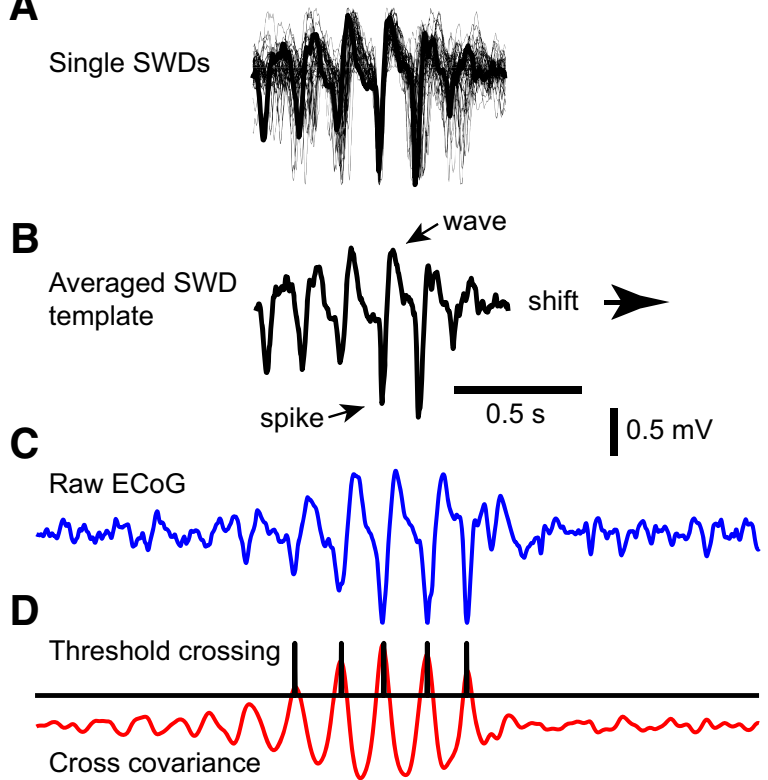

Figure 2. Example of how SWD bursts were automatically detected using an SVM in conjunction with a manually set threshold. $\boldsymbol{A}$, Thirty time-aligned SWD bursts (light traces) in 1 rat with a superimposed average (dark trace) of these traces. $\boldsymbol{B}$, The averaged SWD (spike and wave labeled) was then used as a template and shifted in $2 \mathrm{~ms}$ increments in relationship to the raw ECOG (C). D, At each increment, the cross-covariance between template and ECOG was computed (red trace). A manually set threshold was determined to separate SWD spikes from noise. The peak of spikes crossing this threshold (black trace) identified candidate SWD spikes for SVM analysis.

0.012, respectively). Again, burst durations did not differ between LE and WAG/Rij animals.

To further compare the distributions of SWD burst rates and durations between strains, we replotted the data of Figure 5 as a cumulative probability distribution (the probability a given SWD burst is less than or equal to a specified duration) in Figure 6. A two-sample Kolmogorov-Smirnov test of equality between distributions (Massey, 1951) indicated that the distribution of durations for wild rats did not significantly differ from the BN rats $(\mathrm{D}(12)=0.5: p=0.36)$ but differed from both the LE and WAG/ Rij rats $(\mathrm{D}(12)=0.75: p=0.005$, for both). Differences between distributions of $\mathrm{BN}$ and $\mathrm{LE}$ rats did not reach significance $(\mathrm{D}(12)=0.58: p=0.114)$ but did for WAG/Rij $(\mathrm{D}(12)=0.75$ : $p=0.028)$ rats. LE and WAG/Rij rats did not significantly differ $(\mathrm{D}(12)=0.16: p=1.0)$.

\section{Diurnal rhythmicity of SWDs and associated movement}

An established feature of SWDs in inbred rat strains is diurnal rhythmicity (Van Luijtelaar and Coenen, 1988; Faradji et al., 2000; Smyk et al., 2011). To find out whether wild rat SWDs displayed a diurnal rhythm, we analyzed continuous video/ECoG recording for up to $30 \mathrm{~d}$. All rat groups, including wild rats, displayed increased SWD spike counts (averaged across days) during lights-off compared with lights-on (Fig. 7A). Two-way ANOVA, with one factor representing lights-on versus lights-off and the other factor representing strain, revealed a main effect of light condition $\left(F_{(1,38)}=74.89: p<0.001\right)$ and no significant effect of strain or light-strain interaction. Scaled spike counts for wild rats were nearly double during lights-off $(43.87 \pm 5.49)$ compared with the lights-on $(18.00 \pm 4.21 ; p=0.002)$, a pattern that was similar to $\mathrm{BN}$, LE, and WAG/Rij rats during lights-off $(52.86 \pm 3.72,57.55 \pm 3.04$, and $62.93 \pm 2.94$, respectively $)$ compared with lights-on $(32.01 \pm 4.47,27.21 \pm 4.63$, and $35.56 \pm 3.32 ; p=0.038, p=0.003$, and $p=0.001$, respectively).

To quantify the relative amount of motion during light and dark periods, we derived optical flow from successive frames of associated video captured during ECoG recording. All rats, including wild rats, showed greater motion during lights-off than lights-on (Fig. 7B). This was reflected in two-way ANOVA showing a main effect of light condition $\left(F_{(1,38)}=472.67: p<0.001\right)$. Pairwise analysis of motion in wild, BN, LE, and WAG/Rij rats indicated significant increases during lights-off (77.23 \pm 3.13 , $53.31 \pm 1.85,58.38 \pm 4.09$, and $50.64 \pm 3.03$, respectively) compared with lights-on $(15.21 \pm 1.60,12.39 \pm 0.89,30.69 \pm 2.52$, and $18.34 \pm 2.18 \%$; $p<0.001, p<0.001, p=0.013$, and $p<$ 0.001 , respectively). There was also a main effect of $\operatorname{strain}\left(F_{(3,38)}=\right.$ 14.49: $p<0.001)$ and interaction between light and strain $\left(F_{(3,38)}=\right.$ 18.8: $p<0.001)$. Movement differences between lights-off and lights-on were greater for wild rats $(62.02 \pm 3.12)$ compared with $\mathrm{BN}$ and LE rats (40.01 \pm 1.48 and $27.68 \pm 4.37 ; p=0.001$ and $p<0.001$, respectively). Differences were also greater for $\mathrm{BN}$ rats compared with WAG/Rij rats $(p=0.029)$.

To obtain a more detailed view of diurnal rhythmicity to SWD spike counts and motion, we averaged SWD spike counts and associated optical flow over shorter duration 30 min periods throughout the day and plotted this for each period as the percentage of the daily total (Fig. 8). Cosinor analysis of the resultant SWD spike counts in wild rats showed that, with a period length of $24 \mathrm{~h}$, both one and two cosines (Fig. 8A, red and black, respectively) fit the data. The two-cosine fit was closer to the actual shape of diurnal variation in this example; however, a single cosine fit permitted calculation of the COG (red arrow), facilitating temporal comparisons between strains. As noted by others (DíezNoguera, 2013), the COG, while approximate, is the best parameter of the overall "temporal center," even in cases where the data do not tightly fit a single cosine. In wild rats, the COG corresponded to maximum SWD spike count at a mean time of 21: $09 \pm 0.51 \mathrm{~h}$ (Fig. $8 \mathrm{~A}$, arrow). The peak SWD spike count was during darkness, several hours after lights-out, compared with motion (Fig. $8 B$ ), which was several hours later at $00: 17 \pm 0.35 \mathrm{~h}$ $(p<0.001)$.

Diurnal rhythmicity of SWD spike rates in other groups mirrored that recorded in wild rats. The COG for SWD spike rates occurred near the beginning to middle of the lights-out period for BN, LE, and WAG/Rij rats (Fig. 8C; 20:34 \pm 00:32, $23: 90 \pm 00: 19$, and 21:20 $\pm 00: 19 \mathrm{~h}$, respectively). However, there was a small but significant difference in the COG between groups $\left(F_{(3,24)}=\right.$ 8.9: $p<0.001)$. This was due to an $\sim 3 \mathrm{~h}$ delay in LE rats compared with the wild, BN, and WAG/Rij rats $(p=0.018, p=0.003$, and $p=0.018$, respectively). There were no differences in diurnal timing of SWD spike rates in the other groups. The COG for motion also occurred near the beginning to middle of the lightsout period for $\mathrm{BN}, \mathrm{LE}$, and WAG/Rij rats (Fig. $8 D ; 23: 08 \pm 00: 08$, 22:83 $\pm 00: 46$, and 20:83 $\pm 00: 04 \mathrm{~h}$, respectively). There was a significant difference in the COG for motion between groups $\left(F_{(3,24)}=14.89: p<0.001\right)$. These differences were greater than for SWDs and significant for wild-BN, wild-WAG/Rij, and BNWAG/Rij ( $p=0.003, p<0.001$, and $p<0.001$, respectively). While the average COGs for both SWDs and motion occurred near the beginning to middle of the lights-out period for both SWDs and motion, shifts in the diurnal timing of maximum SWD spike rates did not correlate to the timing of peak motion $(r=-0.008, p=0.97)$. 
A

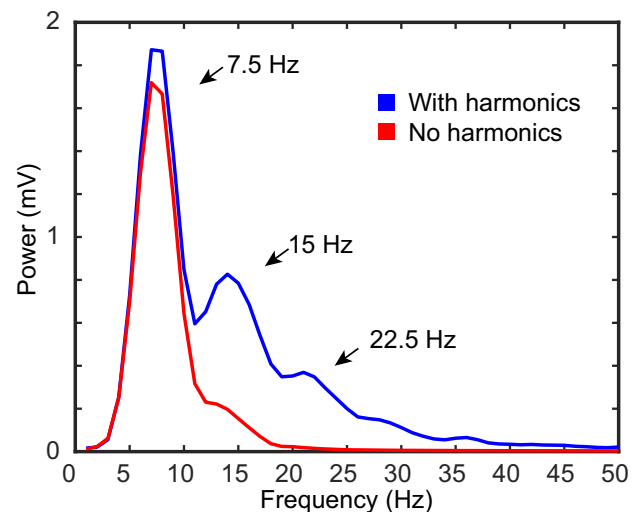

B

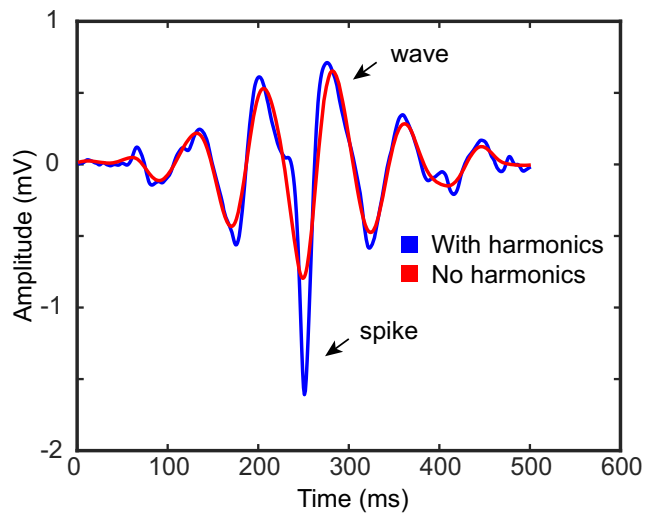

Figure 3. An example of the temporal and spectral features of SWD bursts in a wild rat. $A$, The averaged power spectrum of 1 rat (blue trace; plotted as $m V$ instead of $m V^{2}$ for direct comparison with the amplitude of associated time series) revealed harmonic peaks at $7.5,15$, and $22.5 \mathrm{~Hz}$. When smoothed to attenuate harmonics, only the fundamental frequency of $7.5 \mathrm{~Hz}$ remained. $\boldsymbol{B}$, An inverse FFT of the smoothed spectrum resulted in a simple sinusoidal waveform (red trace) reflecting the wave component of the SWD without the spike component (blue trace).
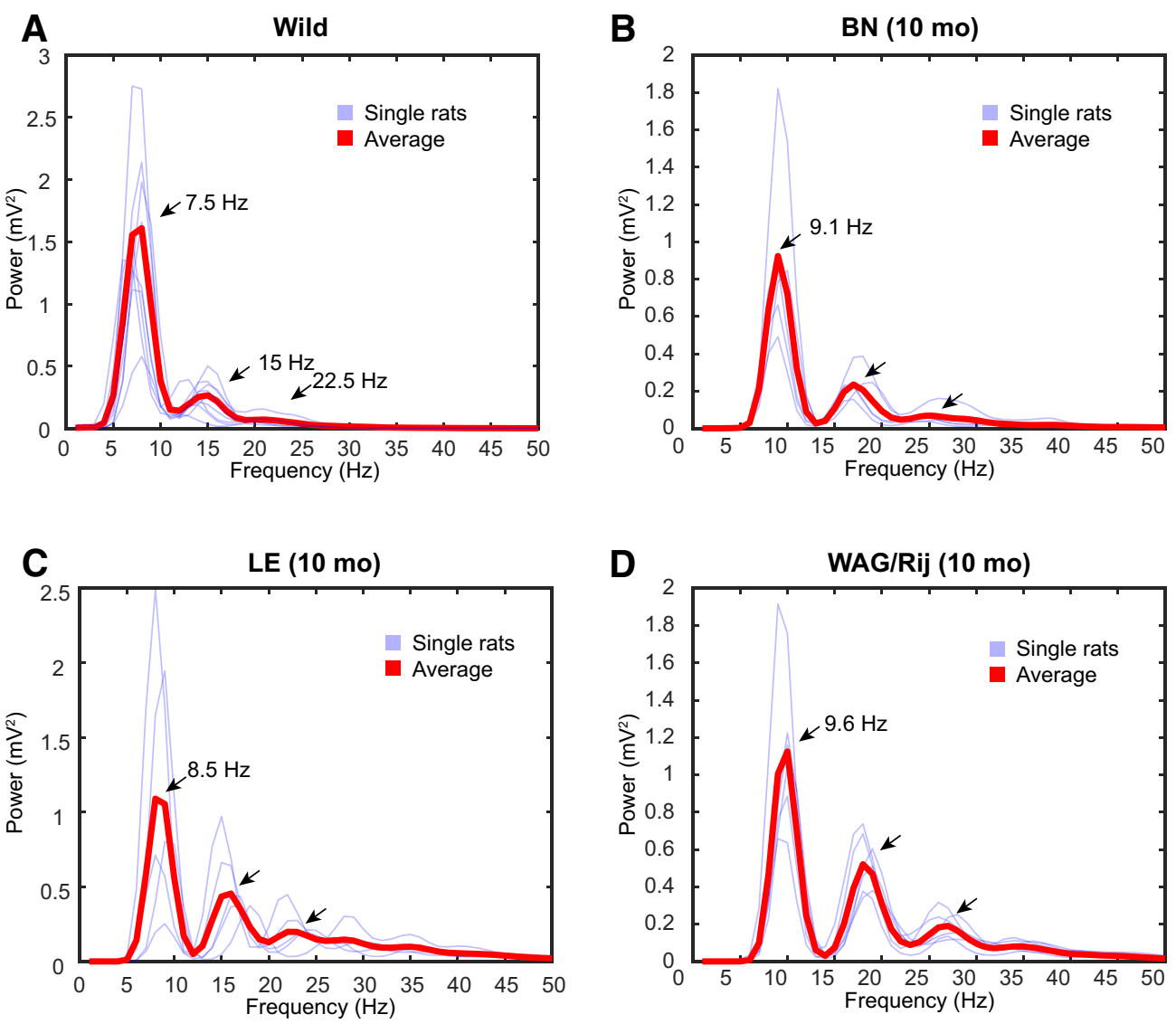

Figure 4. Frequency spectrum of SWD bursts in wild-caught (Wild; $n=9), \operatorname{BN}(n=5), \mathrm{LE}(n=5)$, and WAG/Rij $(n=6)$ rats. Light blue traces represent the frequency spectrum for individual rats, which together produced an average frequency spectrum for the group (red trace). $\boldsymbol{A}$, Wild rats had a center frequency peak at $7.5 \mathrm{~Hz}$, along with harmonic peaks at 15 and $22.5 \mathrm{~Hz}$ in their average frequency spectrum. $\boldsymbol{B}-\boldsymbol{D}$, Ten-month-old BN, LE, and WAG/Rij rats average frequency spectrums displayed center frequency peaks at $9.1,8.5$, and $9.6 \mathrm{~Hz}$, respectively, and associated harmonics. Comparison of average center frequencies revealed that wild rats were $\sim 1.5 \mathrm{~Hz}$ slower than BN and WAG/Rij rats $(p=0.003$ and $p=0.006$, respectively). There were no other significant differences in fundamental frequencies between the strains.

\section{SWDs and immobility}

Cosinor analysis indicated a peak in SWD spike rates during the active dark period but was not of sufficient temporal resolution to establish whether rats were nonetheless immobile during the brief SWD events. Figure $9 A$ shows an example of changes in optical flow (blue trace) in relation to 7 successive SWD bursts (red trace) over several minutes in a WAG/Rij rat. It was clear that movement only occurred between bursts and the rat remained immobile during the bursts. The first 4 bursts showed a distinct cessation of movement at burst onset. The sudden transition from movement to immobility during SWDs appeared as "behavioral arrest" where ongoing activity was suddenly interrupted during the burst. However, this depended on whether there was ongoing activity when the SWD occurred. Often our chronically 
A

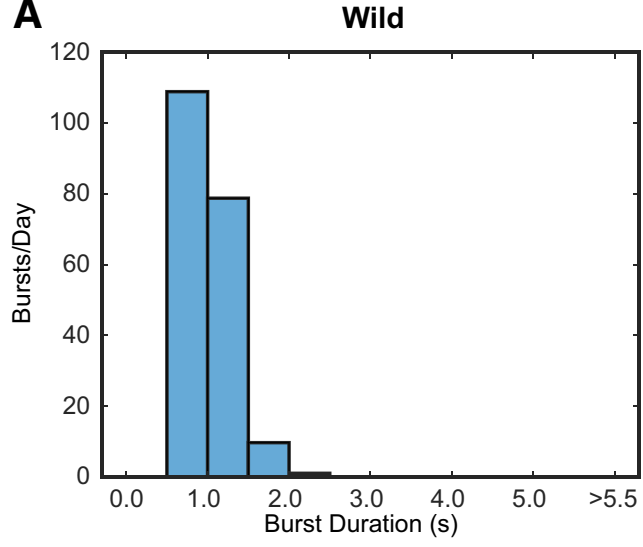

C

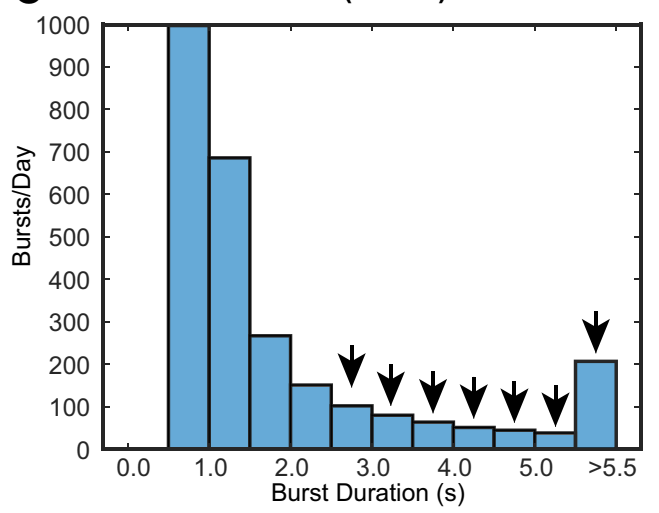

B

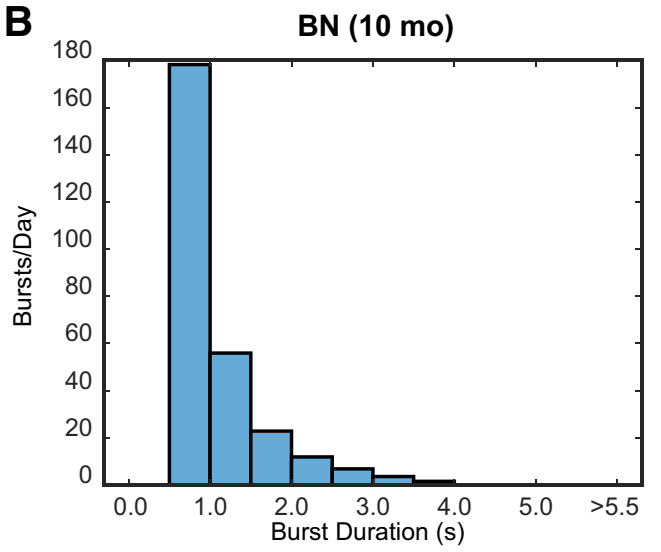

D

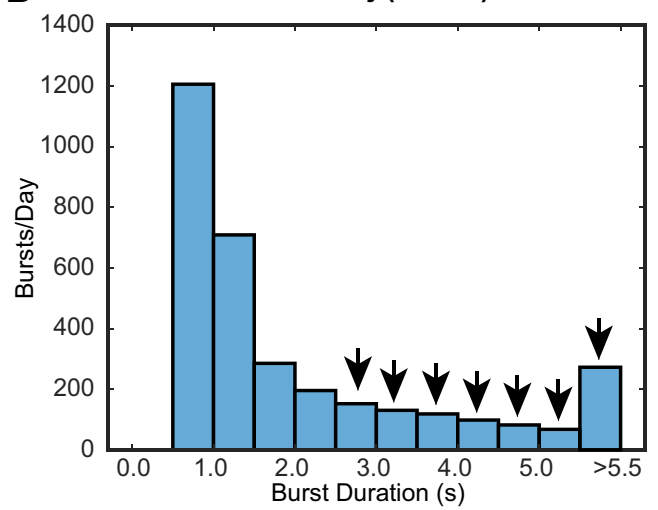

E

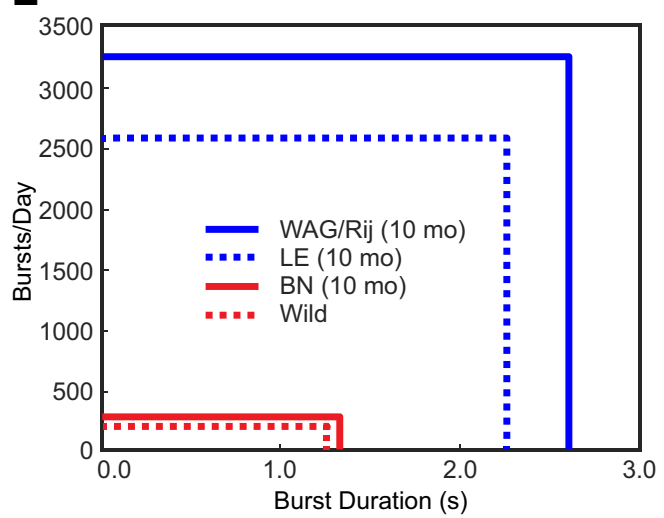

Figure 5. SWD burst rates per burst duration in wild-caught (Wild; $n=9), \mathrm{BN}(n=5), \mathrm{LE}(n=5)$, and WAG/Rij $(n=6)$ rats. $A$, Wild rats averaged $198.7 \pm 83.5 \mathrm{~s} \mathrm{bursts} \mathrm{per} \mathrm{day,} \mathrm{the} \mathrm{majority}$ of which were between 0.5 and 1 slong $(1.25 \pm 0.010$ s). $B$, Ten-month-old BN rats averaged $281 \pm 48.9$ s SWD bursts per day, which were largely comprised of short $0.5 \mathrm{~s}$ long bursts $(1.34 \pm 0.016$ s) but ranged up to 3 or $3.5 \mathrm{~s}$ long bursts. No significant differences were found in SWD burst rates or durations between wild and BN rats. C, D, Ten-month-old LE and WAG/Rij rats displayed significantly higher average burst rates (2683.1 \pm 174.6 and $3380 \pm 180.3$ bursts/d, respectively) compared with wild and BN rats ( $p<0.001$ for both comparisons) but did not significantly differ from each other. Average burst durations for LE $(2.28 \pm 0.014 \mathrm{~s})$ and WAG/Rij $(2.64 \pm 0.015 \mathrm{~s})$ rats did not significantly differ but were significantly longer than wild $(p<0.001$ for both comparisons) and $\mathrm{BN}$ ( $p=0.046$ and $p=0.012$, respectively) rats. $\boldsymbol{E}$, Diagram showing the similarities and differences in average SWD burst rates and burst durations between groups. Wild (dashed red) and BN (solid red) rats were similar in both burst rate and duration. LE (dashed blue) and WAG/Rij (solid blue) were also similar, with more frequent and longer SWD bursts than the wild and domestic $\mathrm{BN}$ rats.

recorded rats showed low activity levels before SWD bursts, resulting in no transition to immobility during the bursts (Fig. 9A, $5-7)$. Figure $9 B$ shows a similar example for a wild rat. While the first SWD burst suggested behavioral arrest, the subsequent 4 bursts revealed only prolonged immobility, recovering after the fifth burst.

Therefore, the most consistent behavioral feature associated with SWDs appeared to be immobility, whether or not this was preceded by ongoing activity. To quantify immobility during SWD bursts, we averaged optical flow from video segments defined by the exact onset and termination of each SWD burst (Fig. 9, dotted traces). Instead of comparing this with optical flow in the seconds just before each burst, which was highly variable, we compared it with the optical flow magnitude averaged across the interburst intervals (Fig. 9, dashed traces). 
A
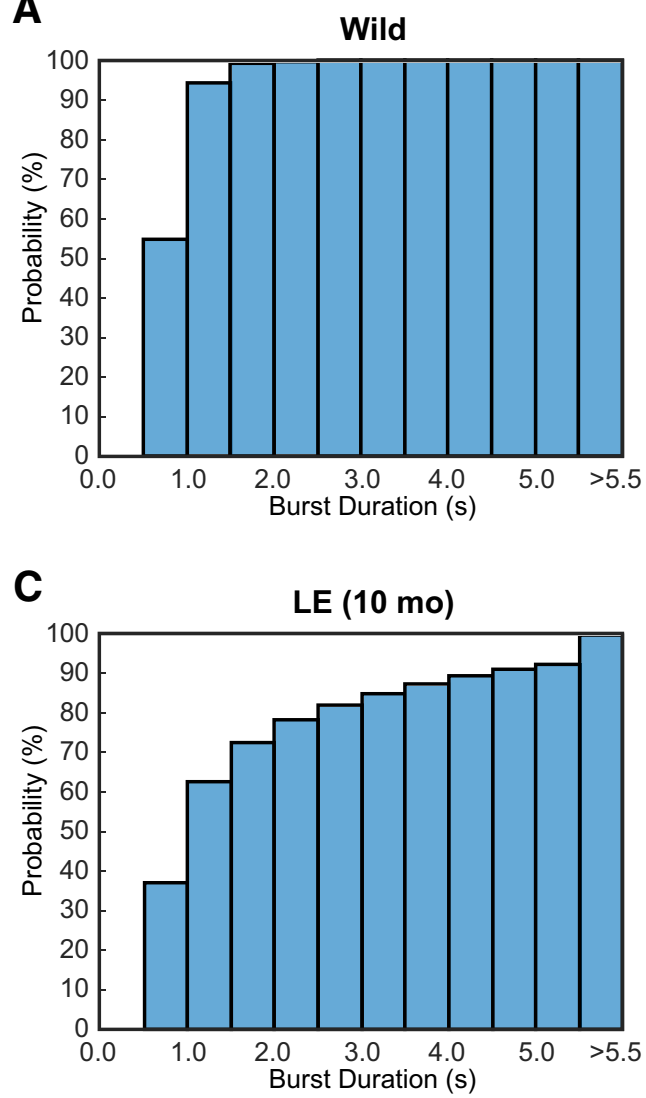

B

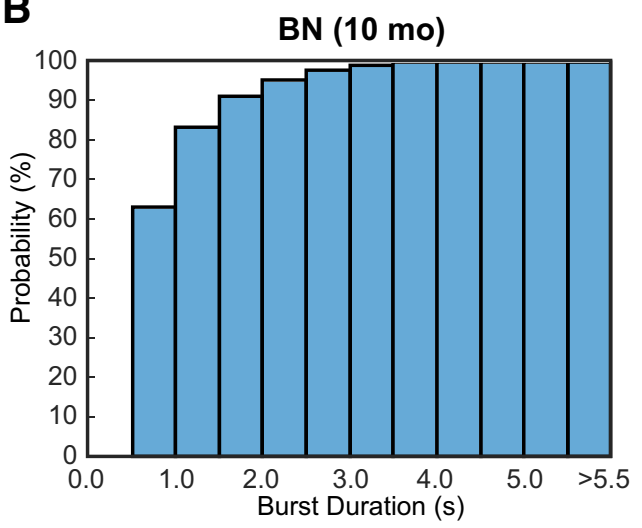

D

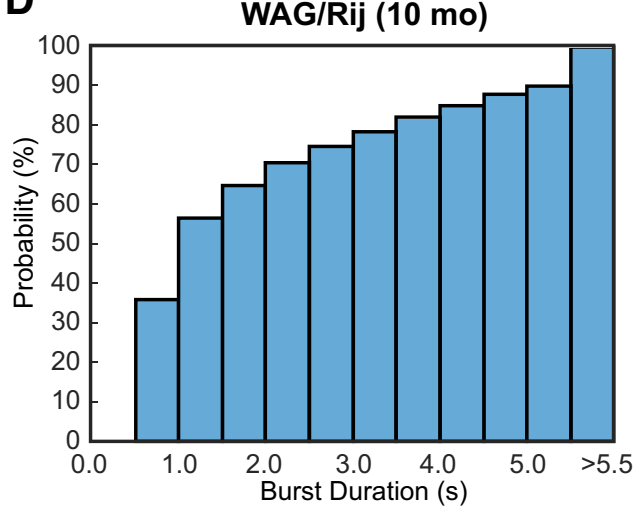

Figure 6. Cumulative probability distributions of SWD burst rates and durations in $(\boldsymbol{A})$ wild-caught (Wild; $n=9),(\boldsymbol{B}) \mathrm{BN}(n=5),(\boldsymbol{C}) \mathrm{LE}(n=5)$, and $(\boldsymbol{D})$ WAG/Rij $(n=6)$ rats. A two-sample Kolmogorov-Smirnov test indicated that the distributions of wild and BN rats did not differ $(p=0.36)$. Nor were there significant differences in the distributions of $L E$ and WAG/Rij rats ( $p=1.0$ ). However, wild rats differed significantly from both LE and WAG/Rij rats ( $p=0.005$ in both cases). The distribution of BN rats also differed from WAG/Rij rats ( $p=0.028$ ) but did not reach significance compared with LE rats $(p=0.114)$.

As shown in Figure 9C, there was almost no movement in wild, BN, LE, and WAG/Rij rats during SWD bursts compared with the movement during interburst intervals. Two-way ANOVA revealed significant differences between movement during SWD versus interburst intervals $\left(F_{(1,36)}=53.3: p<0.001\right)$ but no main effect of strain or interaction. Optical flow for wild rats was nearly 10-fold higher between SWD bursts (Fig. 9C; No-SWD; $53.34 \pm 5.78$ ) compared with during SWD bursts (Fig. $9 C$; SWD; $6.47 \pm 0.72 ; p=0.02)$, a pattern that was similar to $\mathrm{BN}$, $\mathrm{LE}$, and WAG/Rij rats between bursts (51.82 \pm 4.10, $64.34 \pm$ 1.33 , and $43.84 \pm 3.47$, respectively) compared with during SWD $(1.64 \pm .54,2.84 \pm 0.46$, and $3.70 \pm 0.74 ; p=0.001, p<0.001$, and $p<0.001$, respectively).

\section{ETX pharmacology}

ETX administration had a suppressive effect on SWDs in wild, BN, LE, and WAG/Rij rats (Fig. 10), indicating equivalent pharmacosensitivity of SWD bursts across strains. Two-way ANOVA revealed a significant main effect of $\operatorname{ETX}\left(F_{(2,54)}=138.63: p<\right.$ $0.001)$, no main effect of strain, but a significant interaction between ETX and strain $\left(F_{(6,54)}=4.3: p=0.0056\right)$. SWD rates in the $6 \mathrm{~h}$ following ETX injection were significantly reduced compared with the preinjection and postinjection periods $(p<0.001$ in all groups). The interaction between ETX and strain was due to slight but significantly higher post-ETX SWD rates in LE rats compared with wild animals $(p=0.045)$.

\section{Discussion}

Comparison of SWD events in wild-caught rats to outbred and inbred strains

Spectral characteristics

SWD spectra in wild rats mirror absence-like seizures in WAG/ Rij rats and similar events in outbred rats. The center frequencies (7-9 Hz) are similar to those reported by others in WAG/Rij (Coenen and Van Luijtelaar, 2003) and GEARS (Vergnes et al., 1991) rats. Fourier analysis also reveals that SWDs in wild rats are distinguished from other EEG waveforms by the quasiperiodic spike-wave morphology, with the repeating spike resulting in spectral harmonics at multiples of the fundamental frequency. This particular characteristic was also present in our WAG/Rij, outbred $\mathrm{BN}$, and LE rats, and in other inbred and outbred rats (Shaw, 2004, 2007; Meeren et al., 2009; Sitnikova et al., 2009; Taylor et al., 2017). It so uniquely identifies SWDs in GAERS rats that harmonic analysis by itself has been effectively used for automated detection of SWDs (Van Hese et al., 2003, 2009). Based on spectral analysis, we conclude that the electrographic SWD signatures we found in wild rats are a highly similar phenomenon to those found in outbred $\mathrm{BN}$ and $\mathrm{LE}$ rats and in inbred WAG/Rij rats.

\section{Sleep spindles}

These spectral characteristics have also been used to distinguish SWDs from sleep spindles, which are sinusoidal with only a single peak at the fundamental frequency (typically 9-15 Hz) (Meeren 
A

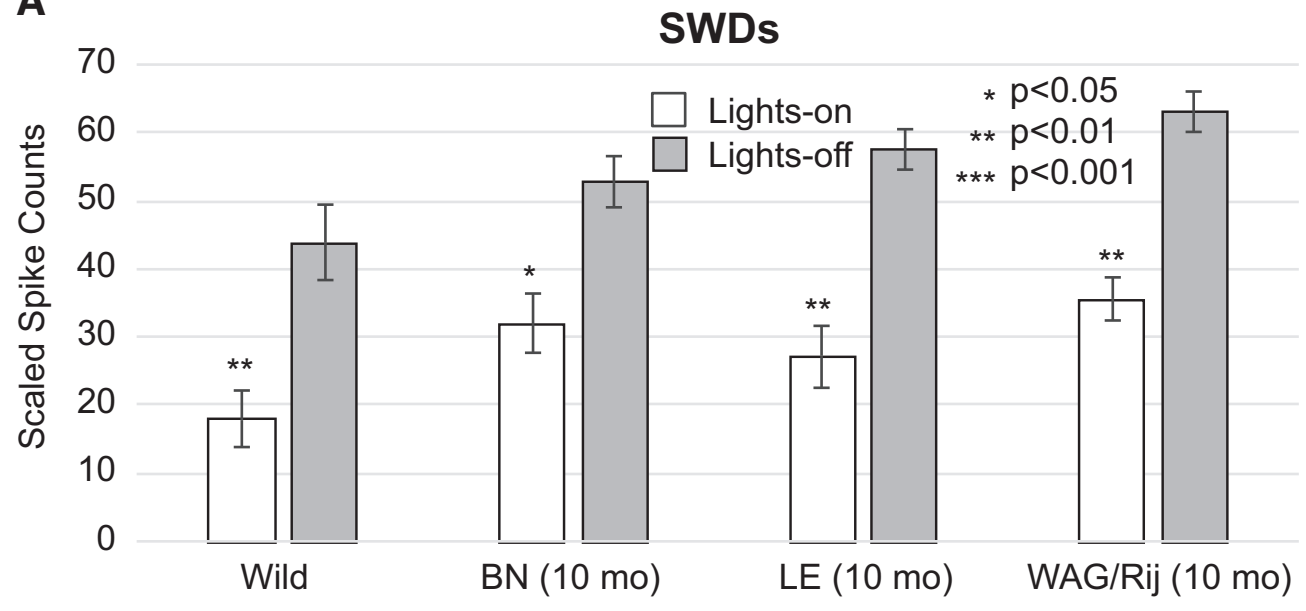

B

Motion

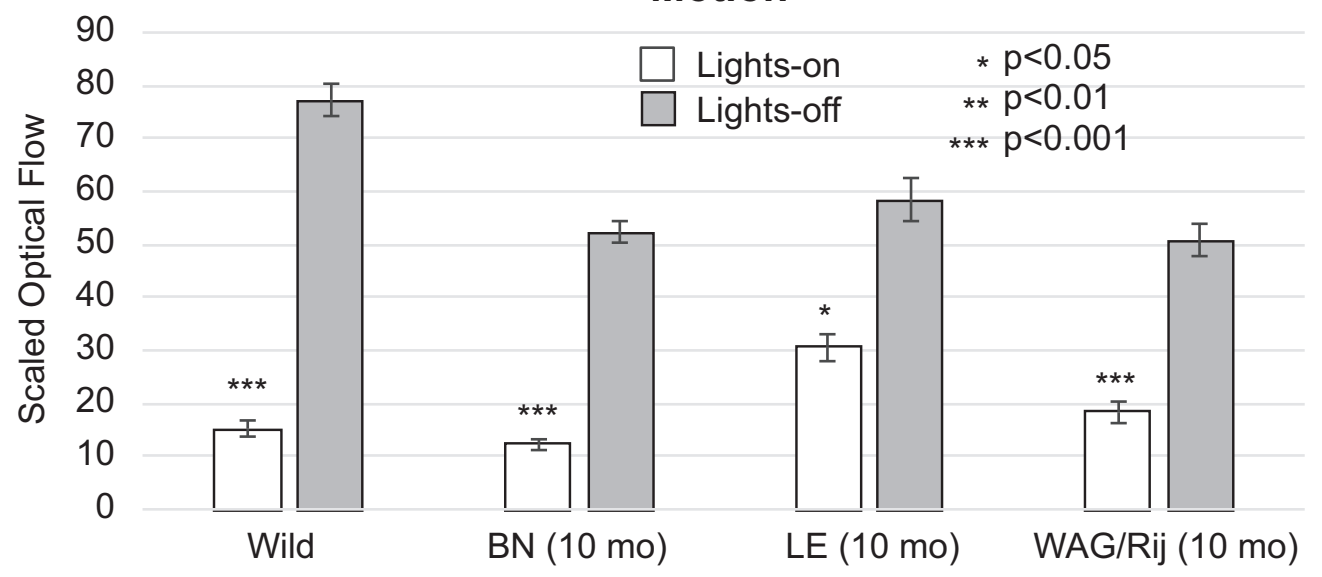

Figure 7. Relationship between SWDs/motion and light cycles in wild-caught (Wild; $n=9), \mathrm{BN}(n=5), \mathrm{LE}(n=5)$, and WAG/Rij $(n=6)$ rats. A, Spike counts (spikes within SWD bursts; scaled to a range of $0-100$ in each rat), which occurred during lights-on and lights-off ( $12 \mathrm{~h}$ light-dark cycle) over the period of chronic recording ( $5-30 \mathrm{~d}$ ). The wild rats were found to have higher spike counts during lights-off than lights-on (43.87 \pm 5.49 and $18.00 \pm 4.21$, respectively; $p=0.002$ ). The 10-month-old BN, LE, and WAG/Rij rats also displayed higher spike rates during lights-off $(52.86 \pm 3.72,57.55 \pm 3.04$, and $62.93 \pm 2.94$, respectively) than lights-on $(32.01 \pm 4.47,27.21 \pm 4.63$, and $35.56 \pm 3.32 ; p=0.038, p=0.003$, and $p=0.001$, respectively). $\boldsymbol{B}$, Relative motion while being chronically recorded via video/ECOG during lights-on and lights-off. This was determined by the magnitude of optical flow recorded by the camera observing a given rat (again, scaled to a range of $0-100)$. All rat groups moved significantly more during lights-off than lights-0n $(p<0.001, p<0.001, p=0.013$, and $p<0.001$, respectively).

et al., 2009; Sitnikova et al., 2009). SWD rates also increase during the dark period, when nocturnal animals are awake and active. Previous reports have noted a diurnal rhythmicity of SWDs in WAG/Rij rats, with a peak of 2-6 h after lights-out (Van Luijtelaar and Coenen, 1988), similar to our maximum SWD rates (3-6 $\mathrm{h}$ after lights-out). SWDs peak at about the same time as the maximum activity level (Fig. $8 C, D$ ), suggesting that they are associated with wakefulness, as noted by others for WAG/Rij rats (Drinkenburg et al., 1991; Marescaux et al., 1992; Smyk et al., 2011).

\section{Immobility}

Immobility of WAG/Rij and GAERS rats is closely time-locked to the onset and duration of the SWD bursts (Vergnes et al., 1991; Marescaux et al., 1992; Coenen and Van Luijtelaar, 2003). Such immobility has typically been considered "behavioral interruption" accompanying SWDs, suggesting nonconvulsive seizures characterizing human absence epilepsy. Optical flow during SWDs shows a highly significant decline, essentially no detectable movement, in all rat strains studied here. As such, it is tempt- ing to state that the SWDs seen in our four groups of rats are absence seizures, as evidenced by the lack of mobility paired with their occurrence. However, a sudden transition from mobility to immobility is only observed with SWD bursts embedded within ongoing activity. We propose that the term "immobility" better describes SWD-related behavior than "behavioral interruption."

ETX

ETX has a powerful attenuating effect on SWDs in inbred rat models of absence epilepsy (Terzioglu et al., 2006; Shaw, 2007; Taylor et al., 2017). Our results indicate that SWDs in wild rats share a similar pharmacosensitivity to ETX seen in both inbred and outbred rat strains. This, however, does not prove that the SWDs in wild rats (or domesticated inbred/outbred rats) are seizures (absence or other); rather, it suggests that the molecular and electrophysiological mechanism(s) responsible for SWDs are shared and preserved despite differences in strain, breeding methods, and environmental origins. 

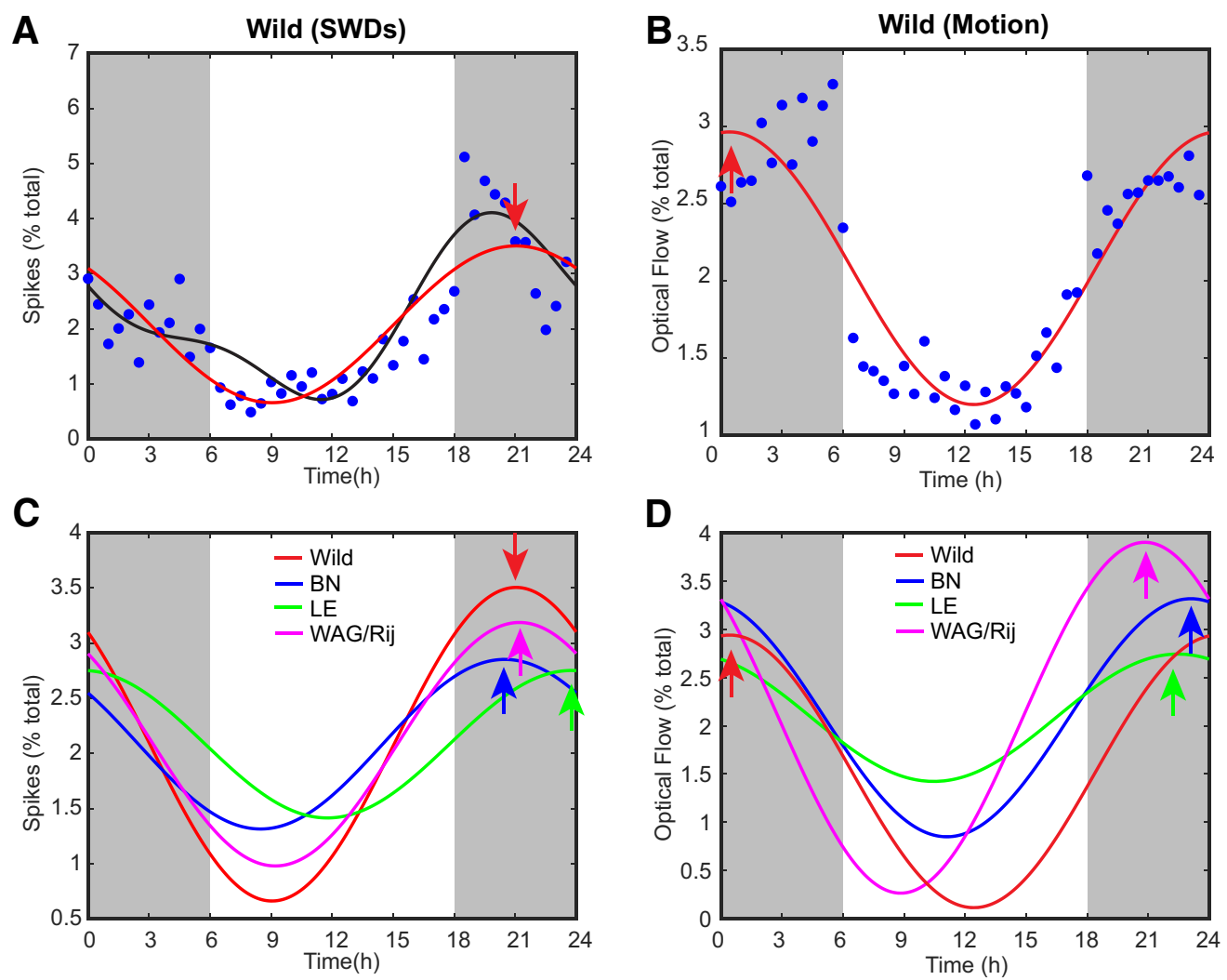

Figure 8. Diurnal rhythmicity of SWD spikes and motion found from chronic video/ECo $\mathrm{G}$ recording $(5-30 \mathrm{~d})$. Cosinor analysis of SWD spike rates and motion were fit with cosine waves with a period length of $24 \mathrm{~h}$ for wild-caught (Wild; $n=9), \mathrm{BN}(n=5), \mathrm{LE}(n=5)$, and WAG/Rij $(n=6)$ rats. Gray represents the lights-off period. White represents the lights-on period. $A$, SWD spike rates occurring over a $24 \mathrm{~h}$ period in wild rats (blue dots) are shown with fits of 1 (red) and 2 (black) cosine waves. While a 2 -cosine fit more closely resembled the shape of diurnal variation in this example, the single cosine fit permitted calculation of $\mathrm{COG}$, which is the best reflection of the overall temporal center. The COG indicated a maximum peak at 21:09 \pm 00:31 $\mathrm{h}$ (during lights-0ff, red arrow). $B$, Wild rat motion (derived from optical flow analysis) had a maximum peak at 0:17 $\pm 00: 21 \mathrm{~h}$ (red arrow) during lights-off. C, Superimposed cosine waves for SWD spike rates for each rat group. Maximum SWD spike percentages for BN, LE, and WAG/Rij rats had corresponding COGs at 20:34 \pm 00:32 h (blue arrow), 23:90 \pm 00:19 h (green arrow), and 21:20 \pm 00:19 h (magenta arrow), respectively. There was a significant $3 \mathrm{~h}$ delay in LE rats compared with the wild, BN, and WAG/Rij rats ( $p=0.018, p=0.003$, and $p=0.018$, respectively). There were no differences in diurnal timing of SWD spike rates in the other groups. $D$, Superimposed cosine waves for motion derived from optical flow analysis. Maximum optical flow percentages for BN, LE, and WAG/Rij rats occurred at 23:08 \pm 00:08 h (blue arrow), 22:83 \pm 00:46 h (green arrow), and 20:83 \pm 00:04 (magenta arrow), respectively. Differences in the COGs for motion were greater than for SWDs and significant for wild-BN, wild-WAG/Rij, and BN-WAG/Rij ( $p=0.003, p<0.001$, and $p<0.001$, respectively). Together, maximums of SWD spike rates and motion occurred during the lights-off condition for all groups of rats throughout the study.

Burst duration and rate

The only distinguishing feature of SWD bursts in wild rats compared with both LE and WAG/Rij rats is shorter $(<2-3 \mathrm{~s})$ and fewer bursts per day. One explanation for this difference could be that the wild rats were younger and therefore produced fewer and shorter SWD bursts because age-related increases in SWDs are widely recognized (Coenen and Van Luijtelaar, 1987; Pearce et al., 2014; Rodgers et al., 2015). However, a more likely explanation is that the difference is strain related. Outbred BN rats, agematched (10 months) to both the LE and WAG/Rij animals, also have significantly fewer and shorter bursts and are nearly identical to our wild rats. This result is consistent with that of Inoue et al. (1990) who found a 10-fold increase in number, and fivefold increase in the duration, of SWD bursts in WAG/Rij compared with BN rats. The fact that our wild and outbred $\mathrm{BN}$ rats have the same burst durations and rates suggests that commercial breeding, and associated environmental differences, such as diet, caging, handling, etc., have a negligible effect on SWDs compared with those in the wild. It also suggests that the order-of-magnitude increases in SWD duration and rate in WAG/Rij absence epilepsy rats are due not just to inbreeding but to strain because these were similar to outbred LE rats.
Are SWDs absence seizures or normal brain rhythms?

Our findings in wild rats would be surprising if SWDs reflected absence epilepsy, and run counter to expectations for natural selection. Selective inbreeding has been used to not only increase the incidence of SWDs in GAERS rats, but also to completely eliminate them in a control strain of Wistar rats (Marescaux et al., 1992). One would expect that natural selection would similarly eliminate SWDs in wild rats if they are absence seizures. We therefore conclude that, in wild rats, SWDs are not seizures but are instead normal brain rhythms (e.g., $\alpha, \mu$, or $\theta$ rhythms) as proposed by others (Semba and Komisaruk, 1984; Kaplan, 1985; Wiest and Nicolelis, 2003; Shaw, 2004). Furthermore, there are no significant differences on any measured parameter between SWDs in wild versus outbred BN rats; domestication does not alter SWDs in this strain. Therefore, we conclude that SWDs are not only normal brain rhythms in the wild, but also presumably in outbred BN rats. Finally, the only significant differences between SWDs of BN versus LE rats were burst durations and rates of occurrence. Yet, these differences appeared to be strain related, and thus do not represent a difference between normal brain rhythms and absence epilepsy. We propose that SWDs in LE rats, and commonly observed in other outbred rat strains used for 

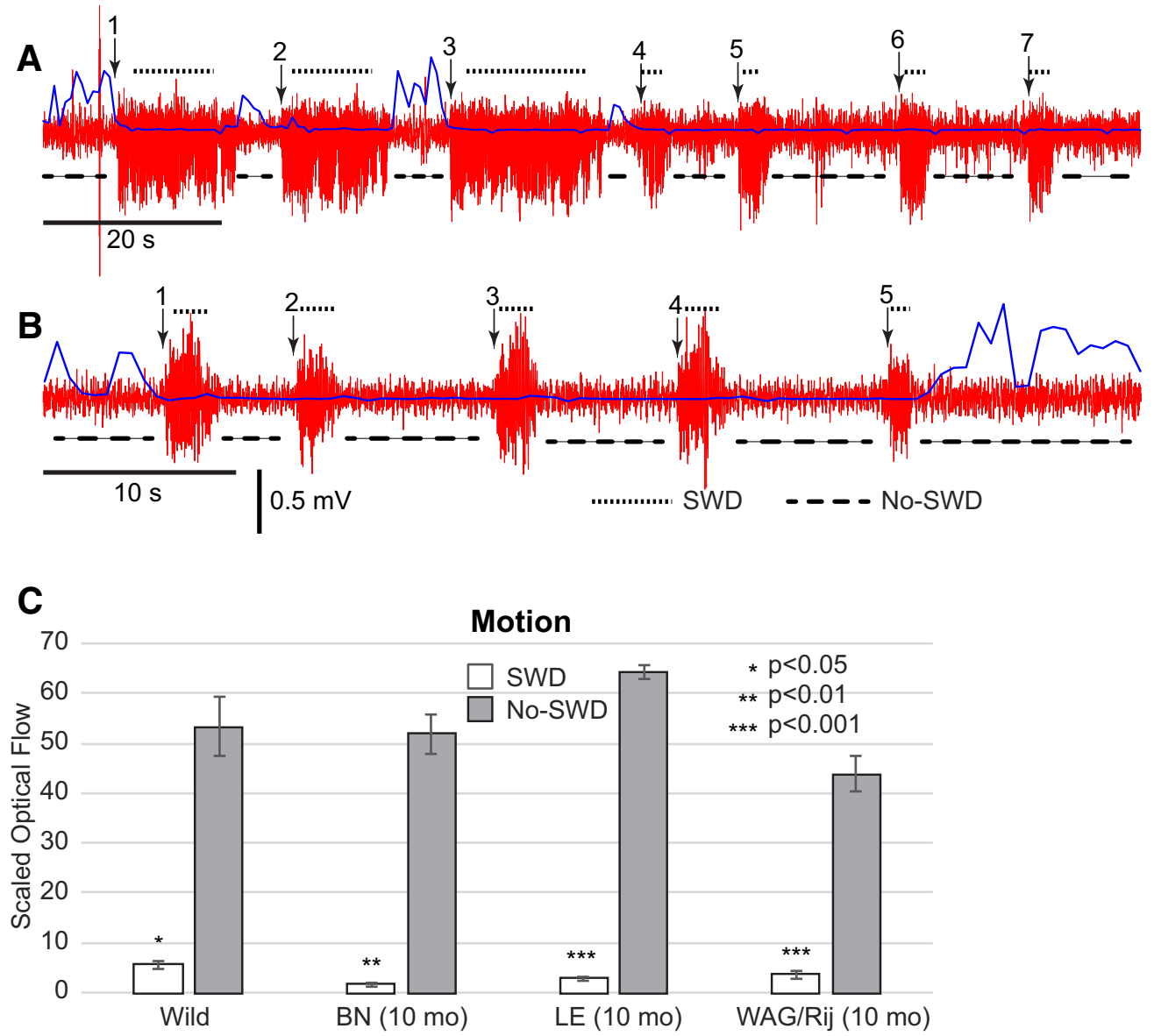

Figure 9. The relationship between SWDs and motion. A, ECoG example of 7 successive SWD bursts (start of each SWD burst signified by arrow and number) in a 10-month-old WAG/Rij rat with a superimposed blue trace representing optical flow occurring during the corresponding video recording. Increases in amplitude of the blue trace indicate increases in optical flow (i.e., movement of the rat). Dotted and dashed lines indicate visually logged periods of SWD burst and interburst epochs, respectively. $\boldsymbol{B}$, Similar SWD burst example and superimposed optical flow for a wild-caught rat. In both examples, although SWD bursts are always associated with immobility, they only occasionally reflect a transition from mobility to immobility (i.e., "behavioral arrest"). C, Comparison of optical flow percentage during SWDs and when no SWDs (No-SWD) were occurring. There was a significantly reduced percentage of optical flow during SWDs in all rats: Wild ( $n=9)$, BN ( $n=5$ ), $\mathrm{LE}(n=5), \operatorname{WAG} / \operatorname{Rij}(n=6) . p=0.02, p=0.001, p<0.001$, and $p<0.001$, respectively.

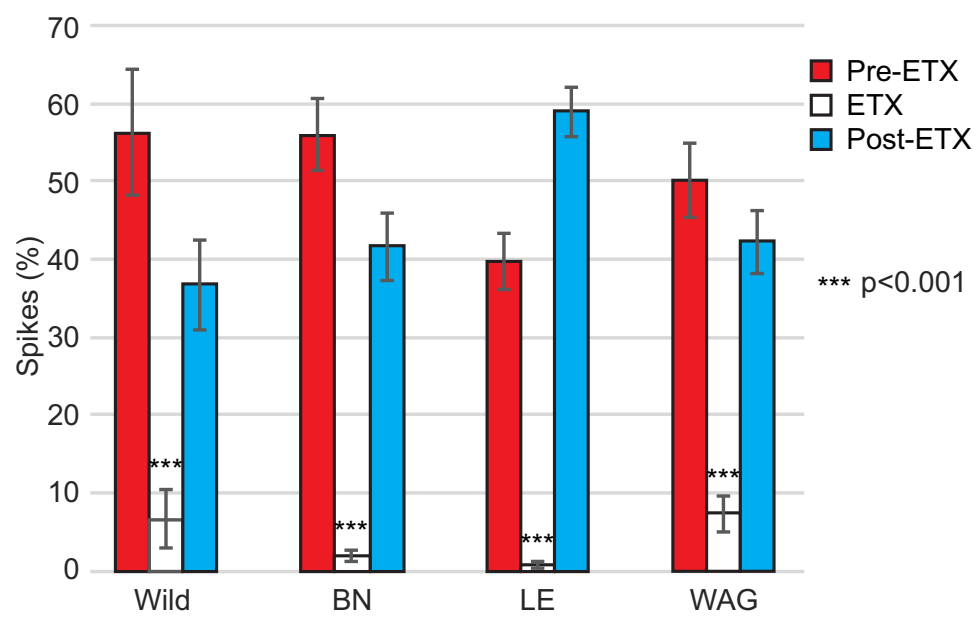

Figure 10. Treatment of SWDs with the antiabsence epilepsy drug ETX in wild-caught (Wild; $n=9), \mathrm{BN}(n=5), \mathrm{LE}(n=5)$, and WAG/Rij (WAG; $n=6$ ) rats. Baseline (Pre-ETX), treatment (ETX), and recovery from ETX (Post-ETX) were sequential $24 \mathrm{~h}$ recordings, of which the first $6 \mathrm{~h}$ (start time $=17: 30 \mathrm{~h}$ ) were analyzed and presented here (plasma concentrations for ETX peak $\sim 2 \mathrm{~h}$ afterinjection). The percentage of SWDs that occurred during ETX was significantly lower compared with Pre-ETX and Post-ETX periods for all rats $(p<0.001$ for all groups). neuroscience research (Pearce et al., 2014), do not reflect absence seizures or a major confound for behavioral studies. Finally, the close similarity between SWDs in LE versus WAG/Rij rats also calls into question whether inbred rats definitively model absence epilepsy.

As a model of human absence seizures, SWDs in inbred rats have been considered to have face validity in that they display simultaneous "clinical" signs of "behavioral arrest" and apparent decreases in responsiveness. SWDs can also be blocked by arousal or mental activities, and are heritable-like human absence epilepsy (Coenen and Van Luijtelaar, 2003). These features, however, might be equally expected if SWDs are associated with normal behavior, such as focused sensory attention, which could be promoted through selective and/or natural breeding. In human absence epilepsy, the discharges are 
substantially slower (classically, " $3 \mathrm{~Hz}$ spike and wave"). Additionally, human absence is typically a juvenile disorder that resolves with age, whereas rat SWDs have not usually been detected until early adulthood and become more prominent with age. Rodent SWDs might still have some predictive validity because they are suppressed by the human absence drug, ETX, a result interpreted as evidence that these discharges are models for absence seizures. However, it is more likely that ETX simply affects certain thalamocortical rhythms, of which human absence seizures are one. Based on our electrophysiological, behavioral, and pharmacological data, we cannot conclude that SWDs in any of our rats are pathological brain rhythms.

The need for valid control studies of SWDs in rats reared in their natural habitat (Kadam et al., 2017) is also essential for judging the epileptic versus nonepileptic nature of similar oscillations reported for rat models of post-traumatic (nongenetic) epilepsy. This includes a model of post-traumatic epilepsy based on fluid percussion injury (D'Ambrosio and Miller, 2010; Dudek and Bertram, 2010; Reid et al., 2016), two models of pediatric epilepsy based on hyperthermia-induced seizures (Dubé et al., 2006), and hypoxia-induced seizures in immature rat pups (Rakhade et al., 2011) that have received scrutiny due to the presence of these ECoG patterns in control (uninjured) rats (Dudek et al., 2010; Pearce et al., 2014; Rodgers et al., 2015).

\section{Future directions}

SWDs and immobility are not definitive signs of epileptic seizures in either inbred or outbred rat strains. An objective and quantitative marker for the transition from normal to epileptic SWDs does not exist. Yet, associated impairment of consciousness is arguably the most important criterion to meet when diagnosing human absence epilepsy (Panayiotopoulos, 2001), and recent human evidence indicates that the extent of impairment can vary between seizures and subjects (Blumenfeld, 2005, 2012). While we have not succeeded in demonstrating impairment of consciousness during SWDs in outbred or inbred rats (Taylor et al., 2017), the present data suggest that this is an essential direction for future development and validation of absence models in rodents. Perhaps the greatest challenge here will be discerning actual impairment of consciousness, in cases where SWDs may reflect absence seizures, from attentional shifts that would be expected if SWDs are instead integral to normal behavior (Wiest and Nicolelis, 2003).

\section{References}

Barron JL, Fleet DJ, Beauchemin SS (1994) Performance of optical flow techniques. Int J Comput Vis 12:43-77.

Berens P (2009) CircStat: a MATLAB toolbox for circular statistics. J Stat Softw 31:1-21.

Blumenfeld H (2005) Consciousness and epilepsy: why are patients with absence seizures absent? Prog Brain Res 150:271-286.

Blumenfeld H (2012) Impaired consciousness in epilepsy. Lancet Neurol 11:814-826.

Booth CJ, Brooks MB, Rockwell S (2010) Spontaneous coagulopathy in inbred WAG/RijYcb rats. Comp Med 60:25-30.

Coenen AM, Van Luijtelaar EL (1987) The WAG/Rij rat model for absence epilepsy: age and sex factors. Epilepsy Res 1:297-301.

Coenen AM, Van Luijtelaar EL (2003) Genetic animal models for absence epilepsy: a review of the WAG/Rij strain of rats. Behav Genet 33:635655.

Crunelli V, Leresche N (2002) Childhood absence epilepsy: genes, channels, neurons and networks. Nat Rev Neurosci 3:371-382.

D'Ambrosio R, Miller JW (2010) What is an epileptic seizure? Unifying definitions in clinical practice and animal research to develop novel treatments. Epilepsy Curr 10:61-66.
Díez-Noguera A (2013) Methods for serial analysis of long time series in the study of biological rhythms. J Circadian Rhythms 11:7.

Drinkenburg WH, Coenen AM, Vossen JM, Van Luijtelaar EL (1991) Spikewave discharges and sleep-wake states in rats with absence epilepsy. Epilepsy Res 9:218-224.

Dubé C, Richichi C, Bender RA, Chung G, Litt B, Baram TZ (2006) Temporal lobe epilepsy after experimental prolonged febrile seizures: prospective analysis. Brain J Neurol 129:911-922.

Dudek FE, Bertram EH (2010) Counterpoint to "What is an epileptic seizure?” By D’Ambrosio and Miller. Epilepsy Curr 10:91-94.

Dudek FE, Ekstrand JJ, Staley KJ (2010) Is neuronal death necessary for acquired epileptogenesis in the immature brain? Epilepsy Curr 10:95-99.

Faradji H, Rousset C, Debilly G, Vergnes M, Cespuglio R (2000) Sleep and epilepsy: a key role for nitric oxide? Epilepsia 41:794-801.

Inoue M, Peeters BW, Van Luijtelaar EL, Vossen JM, Coenen AM (1990) Spontaneous occurrence of spike-wave discharges in five inbred strains of rats. Physiol Behav 48:199-201.

Kadam SD, D’Ambrosio R, Duveau V, Roucard C, Garcia-Cairasco N, Ikeda A, de Curtis M, Galanopoulou AS, Kelly KM (2017) Methodological standards and interpretation of video-electroencephalography in adult control rodents: a TASK1-WG1 report of the AES/ILAE translational task force of the ILAE. Epilepsia 58:10-27.

Kaplan BJ (1985) The epileptic nature of rodent electrocortical polyspiking is still unproven. Exp Neurol 88:425-436.

Marescaux C, Vergnes M, Depaulis A (1992) Genetic absence epilepsy in rats from Strasbourg: a review. J Neural Transm Suppl 35:37-69.

Massey FJ (1951) The Kolmogorov-Smirnov test for goodness of fit. J Am Stat Assoc 46:68.

Meeren HK, Veening JG, Möderscheim TA, Coenen AM, Van Luijtelaar G (2009) Thalamic lesions in a genetic rat model of absence epilepsy: dissociation between spike-wave discharges and sleep spindles. Exp Neurol 217:25-37.

Nandan M, Talathi SS, Myers S, Ditto WL, Khargonekar PP, Carney PR (2010) Support vector machines for seizure detection in an animal model of chronic epilepsy. J Neural Eng 7:036001.

Orrù G, Pettersson-Yeo W, Marquand AF, Sartori G, Mechelli A (2012) Using support vector machine to identify imaging biomarkers of neurological and psychiatric disease: a critical review. Neurosci Biobehav Rev 36:1140-1152.

Panayiotopoulos CP (2001) Treatment of typical absence seizures and related epileptic syndromes. Paediatr Drugs 3:379-403.

Pearce PS, Friedman D, Lafrancois JJ, Iyengar SS, Fenton AA, Maclusky NJ, Scharfman HE (2014) Spike-wave discharges in adult Sprague-Dawley rats and their implications for animal models of temporal lobe epilepsy. Epilepsy Behav 32:121-131.

Posner E (2013) Absence seizures in children. BMJ Clin Evid 2013:0317.

Rakhade SN, Klein PM, Huynh T, Hilario-Gomez C, Kosaras B, Rotenberg A, Jensen FE (2011) Development of later life spontaneous seizures in a rodent model of hypoxia induced neonatal seizures. Epilepsia 52:753765.

Refinetti R, Lissen GC, Halberg F (2007) Procedures for numerical analysis of circadian rhythms. Biol Rhythm Res 38:275-325.

Reid AY, Bragin A, Giza CC, Staba RJ, Engel J Jr (2016) The progression of electrophysiologic abnormalities during epileptogenesis after experimental traumatic brain injury. Epilepsia 57:1558-1567.

Rodgers KM, Dudek FE, Barth DS (2015) Progressive, seizure-like, spikewave discharges are common in both injured and uninjured SpragueDawley rats: implications for the fluid percussion injury model of posttraumatic epilepsy. J Neurosci 35:9194-9204.

Sarkisova K, Van Luijtelaar G (2011) The WAG/Rij strain: a genetic animal model of absence epilepsy with comorbidity of depression. Prog Neuropsychopharmacol Biol Psychiatry 35:854-876.

Semba K, Komisaruk BR (1984) Neural substrates of two different rhythmical vibrissal movements in the rat. Neuroscience 12:761-774.

Shaw FZ (2004) Is spontaneous high-voltage rhythmic spike discharge in Long-Evans rats an absence-like seizure activity? J Neurophysiol 91:6377.

Shaw FZ (2007) 7-12 Hz high-voltage rhythmic spike discharges in rats evaluated by antiepileptic drugs and flicker stimulation. J Neurophysiol 97:238-247.

Sitnikova E, Hramov AE, Koronovsky AA, Van Luijtelaar G (2009) Sleep 
spindles and spike-wave discharges in EEG: their generic features, similarities and distinctions disclosed with Fourier transform and continuous wavelet analysis. J Neurosci Methods 180:304-316.

Smith SJ (2005) EEG in the diagnosis, classification, and management of patients with epilepsy. J Neurol Neurosurg Psychiatry 76 [Suppl 2]:ii2-ii7.

Smyk MK, Coenen AM, Lewandowski MH, Van Luijtelaar G (2011) Endogenous rhythm of absence epilepsy: relationship with general motor activity and sleep-wake states. Epilepsy Res 93:120-127.

Taylor JA, Rodgers KM, Bercum FM, Booth CJ, Dudek FE, Barth DS (2017) Voluntary control of epileptiform spike-wave discharges in awake rats. J Neurosci 37:5861-5869.

Terzioglu B, Aypak C, Onat FY, Kücükibrahimoglu E, Özkaynakci AE, Gören MZ (2006) The effects of ethosuximide on amino acids in genetic absence epilepsy rat model. J Pharmacol Sci 100:227-233.
Van Hese P, Martens JP, Boon P, Dedeurwaerdere S, Lemahieu I, Van de Walle R (2003) Detection of spike and wave discharges in the cortical EEG of genetic absence epilepsy rats from Strasbourg. Phys Med Biol 48:1685-1700.

Van Hese P, Martens JP, Waterschoot L, Boon P, Lemahieu I (2009) Automatic detection of spike and wave discharges in the EEG of genetic absence epilepsy rats from Strasbourg. IEEE Trans Biomed Eng 56:706-717.

Van Luijtelaar EL, Coenen AM (1988) Circadian rhythmicity in absence epilepsy in rats. Epilepsy Res 2:331-336.

Vergnes M, Marescaux C, Boehrer A, Depaulis A (1991) Are rats with genetic absence epilepsy behaviorally impaired? Epilepsy Res 9:97-104.

Wiest MC, Nicolelis MA (2003) Behavioral detection of tactile stimuli during $7-12 \mathrm{~Hz}$ cortical oscillations in awake rats. Nat Neurosci 6:913914 